\title{
COMERCIO SEXUAL, VIOLENCIAS Y RESPONSABILIDAD ESTATAL: MARCO JURÍDICO CHILENO, ¿GARANTE DE DERECHOS O PERPETUADOR DE VIOLENCIAS PARA QUIENES VENDEN SEXO?
}

Sex work, violences and state responsibility: Chilean legal framework, ¿guarantor of rights or perpetuator of violence for those who sell sex?

\author{
Marlene Vera Gutiérrez \\ mgp.vera@gmail.com \\ Universidad de Chile - Chile
}

Recibido: 28-02-2019

Aceptado: 20-05-2019

\section{Resumen}

El comercio sexual se configura como un espacio en el que se circunscriben una serie de violencias. Entre ellas se destacan cuatro focos principales: su construcción histórica como fenómeno socioeconómico, los paradigmas teóricos que polarizan la figura de quienes venden servicios sexuales ya sea como víctimas o como mujeres empoderadas, los modelos jurídicos en los que se inserta y, finalmente, la realidad socio-laboral compleja y precaria de esta industria. A partir de esta realidad múltiple y compleja del comercio sexual, se propone analizar el rol que cumple el Estado chileno en su función de garante de derechos y contrastar este aspecto con las violencias que, a través de su mismo maco regulador, infringe a quienes venden sexo.

Palabras clave: Comercio sexual, modelo jurídico, violencias, prostitución, responsabilidad estatal, Chile.

\begin{abstract}
The sex work is configured as a space in which a series of violence is circumscribed. Among them, I highlight four main focuses: its historical construction as a socioeconomic phenomenon, the theoretical paradigms that polarize the figure of those who sell sexual services as victims or as empowered women, the legal models in which it is inserted and, finally, the complex and precarious socio-labor reality of this industry. Based on this multiple and complex reality of the sex work, I propose to analyze the role played by the Chilean State in its role as guarantor of rights and to contrast this aspect with the violence that, through its own regulatory framework, infringes upon those who sell sex.
\end{abstract}

Key words: Sex work, legal framework, violences, prostitution, state responsability, Chile. 


\section{Introducción}

El presente artículo tiene como objetivo establecer en qué medida el actual modelo jurídico que regula el comercio sexual en Chile se constituye como un protector de derechos fundamentales o un ejecutor de violencia hacia las personas que venden sexo. La pregunta por los factores que participan del ejercicio de violencias por parte del Estado en el comercio sexual surge a partir del reconocimiento de la industria del sexo como un espacio en cuyo centro convergen una serie de violencias (Ribeiro y Sacramento, 2005; Sanders y Campbell, 2007; RedTraSex, 2018).

El comercio sexual ha sido analizado como fenómeno social, antropológico, económico e histórico por diversos autores. Estos estudios se han realizado a partir de dos retóricas principales que lo posicionan ya sea como un espacio laboral de empoderamiento y agencia del uso económico de la propia sexualidad (Oficina Internacional del Trabajo, 1998; Sanders, O’Neill y Pitcher, 2018; Villa Camarma, 2010; Gira Grandt, 2016) o como el resultado del dominio histórico del cuerpo femenino sometido al deseo sexual masculino (Barry, 1987; Bolaños Naranjo, 2008; Díez Gutiérrez, 2009; Lipszyc, 2003; Pateman, 1995). Estas retóricas se encuentran inscritas, además, en determinados momentos históricos y, por lo tanto, imaginarios en torno a "la prostituta" y "la prostitución" que responden a los paradigmas imperantes en cada época (Bell, 1994; Gira Grant, 2016).

Asimismo, los diferentes Estados han adoptado distintos modelos regulatorios con el fin de controlar/eliminar la venta de sexo, generando, de este modo, variados contextos jurídicos para el desarrollo de esta industria, múltiples prácticas permitidas/prohibidas, uso de espacios, condiciones médicas, entre otros factores (Tirado Acero, 2011; Sanders, O’Neill y Pitcher, 2018; Wagenaar, Altink y Amesberger, 2013; Villa Camarma, 2010). Desde esta perspectiva, problematizo la responsabilidad estatal de asegurar/proteger los derechos humanos de los/as ciudadanos/as en contraste con la violencia institucional ejercida hacia quienes venden sexo.

El presente trabajo, entonces, se presenta como un aporte a este amplio campo de estudio que comprende tanto la construcción histórico-teórica del comercio sexual como el contexto jurídico en el que se encuentra inserto, poniendo énfasis en las violencias que se presentan transversales en las dos áreas. A partir de este análisis es posible decir que la violencia hacia las mujeres que venden sexo, considerando la multiplicidad de factores que intervienen en el comercio sexual, es ejercida no solo en su construcción histórica y teórica, sino que también desde el Estado en sus políticas públicas que invisibilizan social y jurídicamente a las mujeres que venden este tipo de servicios.

Desde esta perspectiva es importante relevar que hablar de comercio sexual implica el reconocimiento de una multiplicidad de facetas y contextos en que este se inserta y no de una actividad unidimensional con espacios y formatos establecidos. Así, la venta de servicios sexuales 
puede llevarse a cabo a través de plataformas virtuales, clubes, cafés, prostíbulos, en la calle, en casas de masajes, hoteles o por medio de facilitación de material pornográfico (Weitzer, 2009; Sanders, O’Neill y Pitcher, 2018).

Por su parte, los valores de estos servicios se ven modificados (Musto y Trajtenberg, 2011; Weitzer, 2009) y, por lo tanto, también los tipos de clientes, espacios físicos, recursos, niveles de seguridad/violencia del entorno, entre otros elementos. Estas variables tienen como resultado una industria dinámica, polifacética y de complejo análisis.

Así, a través del análisis cualitativo (Monje Álvarez, 2011) de los datos, contextos e ideas expuestas en este artículo y de un ejercicio deductivo será posible responder a las preguntas acerca de las violencias que intervienen, desde distintas aristas, en la industria del sexo a nivel general y, particularmente en Chile.

En este proceso, el uso del método descriptivo cobra relevancia en tanto es preciso realizar la descripción tanto de procesos históricos como realidades sociales y jurídicas particulares para dar respuesta a las preguntas principales de este escrito.

Ante este panorama, en este artículo se argumenta que, para abordar la temática de la violencia en relación con el comercio sexual, es necesaria una mirada crítica capaz de identificar e incorporar en el análisis tres elementos:

(1) la construcción histórica del comercio sexual,

(2) la amplia gama de retóricas que se levantan en torno a esta industria ${ }^{1} \mathrm{y}$

(3) las distintas posturas jurídicas que la regulan.

Así, en la primera sección de este artículo se expone la construcción y teorización del comercio sexual a lo largo de la historia.

En la segunda parte se analiza cómo este fenómeno se regula de manera estatal y se enmarca en diversos modelos jurídicos que delimitan sus prácticas. En la tercera sección el análisis se centra en saber cómo la venta de sexo se inserta en el contexto jurídico chileno y cuáles son las principales medidas regulatorias tomadas en torno a esta industria.

En la cuarta sección se ponen en tensión las retóricas latentes en las políticas públicas chilenas con las violencias a las que se ven expuestas las personas que venden sexo. De esta manera quedarán en evidencia los diversos tipos de violencia que se circunscriben como realidades para las personas que participan de la comercialización de servicios sexuales.

\footnotetext{
${ }^{1}$ Se han considerado, en este trabajo, los discursos de las mismas trabajadoras sexuales manifestados por medio de sindicatos, agrupaciones y publicaciones como parte de las retóricas que debaten en torno a la industria del sexo y sus participantes.
} 


\section{Construcción histórico-cultural de la venta de servicios sexuales}

Usualmente se relaciona a las prostitutas ${ }^{2}$ con la atemporalidad a través de frases como "el trabajo más antiguo del mundo". Así, el comercio sexual se posiciona en un espacio temporal indefinido de inicio, impidiendo el análisis de la construcción histórica y simbólica del mismo a través de los años y su relación con las prácticas culturales de las sociedades en que se lleva a cabo ${ }^{3}$.

La construcción histórica de la imagen de "la prostituta" ha pasado por diversos procesos de adecuación culturales y lingüísticos. La carga negativa moral, sexual y social que no existía inicialmente, surge en un momento específico de la historia y se refuerza a través de retóricas, políticas públicas, representaciones hegemónicas, entre otras prácticas políticas y sociales. Asimismo, la imagen de trabajadora sexual agente y empoderada se sitúa en un espacio histórico y político en el que las mismas personas involucradas en la venta de sexo instalan sus discursos como parte del debate teórico en torno a la industria del sexo (Bell, 1994; Gira Grandt, 2016; Sanger, 1985).

En la antigua Grecia, tanto la venta de servicios sexuales (en tanto práctica social) como las personas que los entregaban se encontraban fuera del estigma con el que cargan en la actualidad. Existían tres clases de lo que, más tarde, en el siglo XIX, se denominarían como "prostitutas": las dicteriades, las auletrides y las hetairae (Bell, 1994; Licht, 2009; Sanger, 1958). Las primeras entregaban servicios sexuales a las clases más bajas, siendo sus precios adecuados a estos clientes; no obstante, la popularidad con la que contaban era tal, que los ingresos obtenidos eran considerablemente altos (Sanger, 1958). De todos modos, respecto del lugar que ocupaban en el entramado social, estas mujeres compartían posición con las esposas e hijas: "exclusión de la polis y restricción al funcionamiento como medio de intercambio para hombres" (Bell, 1994: 24) ${ }^{4}$. Por su parte, las auletrides dedicaban su labor a las clases medias y desarrollaban sus habilidades en las artes, la música y la danza. Eran, por lo tanto, las invitadas (contratadas) por excelencia a fiestas privadas en donde, sumado a los servicios sexuales, podían tocar flauta, bailar y hacer espectáculos de acrobacias (Licht, 2009). Finalmente, se encontraban las Hetairaes, quienes se posicionaban en

\footnotetext{
${ }^{2}$ En el presente artículo los conceptos son utilizados en relación con la mirada teórica que se esté abordando. Así, las denominaciones "mujer prostituida", "explotación sexual" o "esclavitud sexual" en son aplicadas en exclusivo contexto de retóricas abolicionistas y paradigmas de la opresión/victimización. Por su parte, las palabras "prostituta" y "prostitución" responden a momentos histórico-sociales concretos y no llevan, en este artículo, una carga semántica positiva o negativa, sino que, más bien, se instala en un terreno neutro junto con "comercio sexual", "industria del sexo", "venta de servicios sexuales", entre otros. Finalmente, las palabras "trabajadora sexual" y "trabajo sexual" son conceptos acuñados por las mismas personas que venden sexo y, por lo tanto, decido su inclusión en un ejercicio de visibilización de su postura frente al debate teórico.

${ }^{3}$ Para un análisis de mayor profundidad respecto de la construcción histórica del comercio sexual y de las mujeres que lo llevan a cabo Gira Grant, Melissa (2016): Haciendo de puta. La labor del trabajo sexual (1. ${ }^{\mathrm{a}}$ ed.). Santiago: Pólvora. Y Bell, Shannon (1994): Reading, Writing and Rewriting the prostitute body. Indiana: Indiana University Press.

${ }^{4}$ Traducción propia del original: "Exclusion from the polis and restriction to functioning as the medium of exchange for men".
} 
un nivel social mucho más alto que las otras mujeres en Grecia y desempeñaban importante rol en la vida privada y sexual (Licht, 2009).

De acuerdo con Jess Wells (1982), las Hetaires eran inteligentes, educadas y "las únicas mujeres en la sociedad ateniense con la posibilidad de manejar sus propios asuntos financieros" 5 (Wells, 1982: 6). No solo ofrecían servicios sexuales, sino que participaban activamente en los banquetes y conversaciones masculinas; eran respetadas por sus conocimientos como parteras y como especialistas en hierbas; sabían cómo relacionarse y cautivar a hombres de letras, artistas, generales, entre otros sujetos con poder y conocimiento en la sociedad. Al ser una mezcla perfecta entre "fina intelectualidad y placeres sensuales" (Licht, 2009: 339), los costos de sus servicios, los espacios en que habitaban y su estilo de vida eran de alto nivel.

Así, en la antigua Grecia, ni la marginalidad ni la estigmatización eran ideas asociadas a la venta servicios sexuales como práctica social. La "otredad" y subalternidad de las mujeres que venden servicios sexuales tiene un origen histórico particular amparado en determinadas retóricas que las convirtieron en sujetos abyectos. La carga moral y criminalización que han adquirido a lo largo de la historia no es "atemporal", sino que responde a una construcción binaria de la "buena/mala mujer", a la mirada dicotómica y simplista de la división esposa/puta instalada en la modernidad (Bell, 1994; Gira Grant, 2016; Sanders, O’Neill y Pitcher, 2018).

Las terminologías y, por lo tanto, la categorización e identificación de los sujetos, juegan un rol importante en el proceso de construcción de "la prostituta". De acuerdo con Melissa Gira Grant (2016), la palabra "prostituta" no existía sino hasta el siglo XVI, época en la que su uso, en la lengua inglesa, era a través del verbo "to prostitute" que decía relación con el acto de poner algo en venta. Por su parte, el término despectivo y con mayor carga negativa "puta" o "whore" tendría su raíz etimológica en el siglo XII a.C., en cuya semántica no se encontraría alojado el imaginario de la venta de servicios sexuales, sino que "whoring refería a las relaciones sexuales fuera del matrimonio y connotaba inmoralidad o promiscuidad sin relación con el dinero. Y la palabra whore era usada para etiquetar a cualquier mujer que excediera los límites de respetabilidad contemporáneos" (Laura María Agustín citado en Gira Grant, 2016: 36).

Así, la estigmatización de la venta de servicios sexuales y la connotación negativa que, aparentemente, se instala como inherente a esta práctica no sería otra cosa que una construcción basada en retóricas hegemónicas que, en determinados momentos históricos, la condenaron.

La modernidad fue un periodo en el que, gracias a los imaginarios imperantes, a la importancia de la moral, el conservadurismo y la religiosidad, el comercio sexual fue fuertemente condenado social, punitiva y moralmente. Particularmente, en el proceso modernizador y proletarizador vivido en Chile, las mujeres que llevaban a cabo la venta de servicios sexuales eran consideradas como un problema sanitario y de orden público. Los paradigmas bajo los cuales se

\footnotetext{
${ }^{5}$ Traducción propia del original: "the only women in Athenian society allowed to manage their own financial affairs".
} 
ordenaba el cuerpo social el Chile durante el siglo XIX y XX eran fundamentalmente conservadores e higienistas (Folchi Donoso, 2007; Góngora Escobedo, 1999). Estas miradas entregaban una imagen hegemónica construida en torno a la venta de sexo que posicionaba a los participantes de esta industria (principalmente a las prostitutas) como sujetos parias en la sociedad, marginalizados y criminalizados.

Desde un punto de vista conservador, "La Mujer" debía responder con un alto estándar moral (Urriola Pérez, 1999; Vera Gajardo, 2016; Zárate, 1995), dedicándose de manera sumisa y devota a la familia, los hijos y las tareas de reproducción y cuidado ya fuera como dueña de casa o como trabajadora en una fábrica. Las mujeres que vendían servicios sexuales, por lo tanto, se encontraban en una posición de desacato moral a las reglas de correcto comportamiento social de la época. Por su parte, desde la mirada higienista, las prostitutas representaban el mayor foco infeccioso de enfermedades venéreas (Maira, 1887; Staforelli, 1925) y, por lo tanto, la necesidad de controlar sus prácticas y los espacios que habitaban eran de importancia sustancial para la salud pública (Araya Ibache, 2006; Durán, 2009; Góngora Escobedo, 1999).

Frente al desacato social y moral que representaban las mujeres que vendían sexo y al problema sanitario que significaba la proliferación y aumento de las enfermedades venéreas, la figura de "la prostituta" se criminalizó. La imagen hegemónica levantada en torno a la palabra "prostituta" se llenó de connotaciones negativas que tenían relación con infecciones venéreas, mal vivir, inmoralidad, entre otras características que hicieron, de las mujeres que vendían sexo cuerpos abyectos en situación delictual y en constante conflicto legal.

Posteriormente, y a partir de los primeros años del siglo XX, los debates feministas modificaron la mirada en torno al comercio sexual y a los participantes de esta industria. La "puta marginada", en cuya representación hegemónica se encontraban alojadas todas las connotaciones criminalizadoras, se convierte en "prostituta victimizada" (Gira Grant, 2016: 35), esclavizada sexualmente y violentada por el sistema patriarcal capitalista que somete y mercantiliza su cuerpo (Pateman, 1995; Barry, 1987; Bolaños Naranjo, 2008; Lipszyc, 2003). A partir de esta línea de pensamiento la antigua "prostitución" se entiende ahora como "esclavitud sexual" y a las "prostitutas" pasan a ser "mujeres abusadas" por un sistema que la oprime sexualmente.

Las discusiones en torno a la venta de sexo recibió, a partir de 1970, una nueva perspectiva: la de las trabajadoras sexuales (Sanders, O’Neill y Pitcher, 2018; Gira Grant, 2016). A través de distintas organizaciones, sindicatos o colectivos ${ }^{6}$, las trabajadoras sexuales han relevado el carácter laboral de la industria del sexo, por un lado, y han denunciado, por otro lado, la desprotección sociojurídica en la que se encuentran. Por su parte los términos "trabajadora sexual" y "trabajo sexual" son acuñados por las mismas trabajadoras y contribuyen a incluir una nueva mirada al fenómeno de la industria del sexo.

\footnotetext{
${ }^{6}$ Algunas organizaciones de trabajadoras sexuales son: Fundación Margen, Fundación Amanda Jofré, Red TraSex, Sex Workers Alliance Ireland, Asociación de Mujeres Meretrices de Argentina, Sex Workers Europe, entre otras.
} 


\section{Comercio sexual: una industria transgredida por múltiples violencias}

El comercio sexual, por lo tanto, no es un fenómeno unidimensional, sino que se presenta complejo y múltiple en diversos aspectos (teóricos, históricos, políticos, culturales, etc.); no obstante, en el presente texto se busca relevar que existe un elemento que se instala de manera transversal dentro de esta multidimensionalidad: la violencia.

A continuación, se enumera y describe cómo estas violencias han sido ejercidas sobre las personas que venden sexo tanto desde una perspectiva histórica como desde la concreción de políticas públicas que regulen la industria sexual.

Así, desde un punto de vista histórico, la construcción misma de la figura de las trabajadoras sexuales no se ha visto exenta de violencias (Bell, 1994). La inicial criminalización y posterior victimización de las mujeres dedicadas al comercio sexual ha resultado en dos grandes consecuencias: en primer lugar, el refuerzo de una mirada estigmatizada y binaria respecto de la venta de sexo, reduciendo este complejo fenómeno a dos visiones polarizadas (criminalidad/opresión) (Weitzer, 2009). En segundo lugar, se eliminan de esta construcción todos los otros roles y características de las mujeres que ejercen el comercio sexual (madres, trabajadoras, estudiantes, migrantes, parejas, entre otras) y se resalta solamente aquello que parece ser esencialmente identitario y definitorio: "ser prostituta" (Gira Grant, 2016).

Específicamente, durante el siglo XIX en Chile las mujeres que ejercían la prostitución como fuente de ingreso económico fueron acusadas de criminales, de cometer actos delictuales e inmorales, de ser un foco infeccioso para la sociedad, entre otros modos de discriminación y estigmatización (Góngora Escobedo, 1999; Gálvez Comandini, 2014; Zárate, 1995; Maira, 1887). Convertidas en sujetos abyectos fueron violentadas y excluidas a causa de la construcción hegemónica de imagen de "la prostituta" levantada desde las élites político-higienistas.

No obstante, la criminalización y condena moral no serían la única fuente de violencia a la que se verían enfrentadas las mujeres que vendían servicios sexuales en tiempos decimonónicos. La condición precaria de los distintos lupanares, los malos tratos recibidos por parte de los regentes, la precariedad de alimentación y vestuario, la obligación de entregar servicios sexuales aún enfermas, entre otros factores, convertían estos espacios laborales en sitios donde se encontraban múltiples formas de violencia dirigidas hacia estas mujeres (Gálvez Comandini, 2014; Vera Gutiérrez, 2018).

La violencia en el comercio sexual, por tanto, no solamente es una práctica que se ha ejercido de manera histórica y sistemática a lo largo de su construcción, sino que es también una característica concreta de la realidad que experimentan las trabajadoras sexuales en los distintos espacios en que ejercen su labor. Los malos tratos a los que se veían expuestas las mujeres en los prostíbulos del siglo XIX no difieren del difícil contexto al que se ven enfrentadas en la actualidad (OMS, 2013; RedTraSex, 2013). La actual invisibilización jurídica (que se hace patente a través de desprotección policial, vulnerabilidad social e estigmatización sanitaria) juega un importante rol a la 
hora de posicionar a las personas que venden sexo en espacios laborales seguros. Las violencias, entonces, se multiplican: la estigmatizada construcción histórica y teórica que las marginaliza, los riesgos y precariedades del ejercicio de una labor criminalizada, y la desprotección social, jurídica y policial se configuran como un tejido de violencias en torno al comercio sexual y a quienes lo ejercen (Scoular y O’Neill, 2007; Ribeiro y Sacramento, 2005; Villa Camarma, 2010).

Las condiciones sociales, laborales, jurídicas o sanitarias del ejercicio del comercio sexual han sido relevadas por los discursos y perspectivas de las mismas trabajadoras sexuales. En la actualidad, se han agrupado en distintas redes, sindicatos y organizaciones nacionales, como Fundación Margen y Fundación Amanda Jofré (Chile), o internacionales como la Red de Mujeres Trabajadoras Sexuales de Latinoamérica y el Caribe (RedTraSex) y Sex Workers Europe. La nueva perspectiva aportada por las trabajadoras sexuales deja en evidencia los múltiples focos de violencia de los que son víctimas (Gira Grandt, 2016; Sanders y Campbell, 2007).

En primer lugar, y de acuerdo con la Guía de buenas prácticas en el personal de sistema de salud "al no estar regulado, el trabajo sexual es ejercido en condiciones muy precarias" (RedTraSex, 2013: 7). Los encuentros pueden darse en hoteles, calles, saunas, bares entre otros espacios con distintas condiciones de seguridad para las mujeres. La elección de trabajar de manera independiente $\mathrm{y}$, por lo tanto, obtener mayores beneficios económicos de la transacción es inversamente proporcional al nivel de seguridad que las trabajadoras sexuales podrían encontrar en un hotel o en "un boliche [en donde] te sacan el 50\% de tu trabajo" (RedTraSex, 2013: 7).

Por su parte, el informe titulado Trabajo sexual y violencia institucional: vulneración de derechos y abuso de poder hacia mujeres trabajadoras sexuales (RedTraSex, 2016) destaca un segundo tipo de violencia: policial. Exponen que, dentro de las situaciones discriminación a las que se enfrentan, se encuentran el abuso de poder policial, la solicitud de servicios sexuales gratuitos por parte de fuerzas de seguridad, detenciones arbitrarias, intimidación, acoso, extorsiones, entre otras (RedTraSex, 2016: 7). En el informe mencionado es posible encontrar, además, una serie de declaraciones y testimonios narrados por las víctimas de estas prácticas, mostrando, de manera concreta, los altos niveles de vulnerabilidad de las trabajadoras sexuales frente a las autoridades policiales.

En tercer lugar, y desde un punto de vista sanitario, quienes venden servicios sexuales se ven expuestos a la constante estigmatización y discriminación en los servicios de salud a los que asisten. Así, en la Guía de buenas prácticas en el personal del sistema de salud las trabajadoras sexuales dan cuenta de la violencia de género y discriminación que deben recibir: recibir miradas hostiles, términos humillantes o descalificadores, ser tratadas como focos de infección venérea, no recibir atención de salud sexual integral, entre otras prácticas (RedTraSex, 2013: 11-14).

Finalmente, y desde una perspectiva jurídica, la invisibilización de la realidad del comercio sexual y la ausencia de políticas públicas que regulen esta industria y entreguen seguridad tanto a trabajadoras como a clientes se constituyen como un espacio de violencia institucional hacia este grupo socio-económico. 
Las mujeres que venden servicios sexuales, entonces, se han visto violentadas desde las distintas retóricas que las construyen, teorizan, estigmatizan y marginalizan. Una de ellas es la construcción hegemónica de una imagen unidimensional que las posiciona en la categoría de criminal, víctima de explotación sexual o mujer empoderada (Sanders, O’Neill y Pitcher, 2018; Weitzer, 2009). La propuesta, entonces, consiste en ampliar la mirada en torno al fenómeno del comercio sexual y reconocerlo en sus distintos contextos: comprender que existe tanto ejercicio de agencia como determinismo en la decisión de vender sexo y, para esto, dar espacio a los discursos de las trabajadoras sexual quienes, en primera persona, pueden dar cuenta de su realidad; reconocer las condiciones precarias y violentas que esta labor implica, pero, al mismo tiempo, incluir en el análisis la multiplicidad de formatos y situaciones de venta de servicios sexuales, que vuelven a la industria dinámica y compleja; finalmente, relevar la responsabilidad estatal en las múltiples violencias ejercidas hacia el comercio sexual y la necesidad tanto de dar protección a quienes venden sexo de manera forzada y esclavizada como de dar seguridad laboral, social, sanitaria y policial a quienes permanecen ejerciendo el trabajo sexual de manera agente. Puesto que

“Aunque la prostitución sea entendida como un producto de la desigualdad social estructural entre hombres y mujeres, esto no significa que haya que mirar hacia otro lado y no reconocer su existencia ni las condiciones sociales y laborales en que se desarrolla ni las reivindicaciones de los derechos de estas mujeres" (Villa Camarma, 2010: 174).

\section{Modelos jurídicos y responsabilidad estatal en la protección de los derechos humanos}

Frente a la trascendencia temporal, al dinamismo y adaptación del comercio sexual, los poderes políticos han tenido que recurrir a distintas estrategias para regularlo. Los modelos jurídicos adoptados funcionan como instrumentos que norman las prácticas de los sujetos (Foucault, 2003) que, en este caso, se encuentran en condición de parias a causa de la construcción estigmatizada de la venta de sexo (Bell, 1994; Gira Grandt, 2016). Asimismo, estas normas responden a una concepción determinada de cada Estado respecto de la industria del sexo, a una serie de saberes del cuerpo (Foucault, 2012) y a un objetivo regulatorio particular.

Los cuatro principales modelos regulatorios son: abolicionista, prohibicionista, reglamentarista y laborista. Las diferencias entre las distintas posturas jurídicas resulta significativa respecto del nivel de criminalización o aprobación como industria comercial de la venta de sexo (Canales Nettle, 2005; Wagenaar, Altink y Amesberger, 2013; Villa Camarma, 2010; Sanders, O’Neill y Pitcher, 2018). Ahora bien, no todos los sistemas jurídicos responden exactamente a los parámetros de estos cuatro modelos, sino que, además algunos Estados optan por la invisibilización o la regulación mixta o indefinida (Fernández Darraz, 2011), situación que aporta aún más 
heterogeneidad a los ya complejos contextos políticos y sociales en que se inserta la industria del sexo.

El modelo abolicionista surge de las retóricas que consideran que quienes venden sexo lo hacen bajo un sistema de opresión económica que las determina en su decisión y, por lo tanto, es una práctica incompatible con la dignidad humana. Las personas que venden servicios sexuales se configuran, bajo esta mirada, como víctimas de explotación y abuso sexual (Bolaños Naranjo, 2008; Lipszyc, 2003; Pateman, 1995; Díez Gutiérrez, 2009) y, por lo tanto no se penalizan sus prácticas, sino la de aquellos que se constituyen como colaboradores en la opresión: clientes y proxenetas. Bajo el modelo abolicionista se criminaliza tanto la compra como la administración de los servicios sexuales, mas no su venta, puesto que "la prostitución se considera una faceta más de las manifestaciones de la violencia contra las mujeres" (Villa Camarma, 2010: 170).

Entre los países que han adoptado este modelo se encuentran Francia y Suecia. Por su parte, las trabajadoras sexuales francesas (Sputnik mundo, 08-04-2017) y los autores que cuestionan el supuesto éxito de estas medidas en Suecia (Gould, 2002; Dodillet y Östergren, 2011) dan cuenta de los problemas concretos de su aplicación: las transacciones y tiempos para establecer límites con los clientes son menores, por lo que se corren mayores riesgos; las trabajadoras deben acudir a espacios con menos iluminación y seguridad ya que deben evitar exponer a sus clientes; finalmente, afirman que las declaraciones de éxito en la disminución del comercio sexual proclamadas por las autoridades "no resultan respaldadas por los hechos conocidos o por los estudios realizados" (Dodillet y Östergren, 2011: 2).

En segundo lugar, desde la mirada de las retóricas que respaldan el modelo prohibicionista, la transacción mercantil sexo/dinero es considerada "un delito de carácter moral y legal, que provocaría la perdición y corrupción de los valores tradicionales" (Villa Camarma, 2010: 159). Bajo este sistema se penaliza tanto la compra como la venta de sexo, prohibiéndose el comercio sexual en sus múltiples formas. La consideración de la "mujer prostituta" como un "sujeto criminal", define a las mujeres que venden sexo como "malas mujeres" en contraste con aquellas esposas y madres que representan los valores tradicionales y que cumplen con el rol femenino impuesto hegemónicamente (Villa Camarma, 2010; Weitzer, 2009).

Irlanda es uno de los países que ha criminalizado el comercio sexual y prohibido tanto la compra como la venta de este tipo de servicios. Al respecto, las trabajadoras sexuales irlandesas en un informe publicado el año 2017 han declarado que "eliminar las sanciones penales es esencial para combatir el estigma y permitir que las trabajadoras sexuales sean visibles y participen en las leyes desarrolladas para protegerlas" (Sex Workers Alliance Ireland, 2017) ${ }^{7}$.

En tercer lugar, el modelo reglamentarista busca regular el comercio sexual no a través de la prohibición absoluta, sino de la delimitación de las prácticas y espacios permitidos para llevarlo a

\footnotetext{
${ }^{7}$ Traducción propia del original: "Removing criminal sanctions is essential to tackling stigma and enabling female sex workers to be visible and participate in the laws developed to protect them".
} 
cabo. Las retóricas reglamentaristas reconocen "la prostitución como un mal social que, al no poderse combatir, debe ser regulado frente a los efectos perniciosos relacionados con la salud, la convivencia y las buenas costumbres" (Tirado Acero, 2011: 139). Este modelo busca, por lo tanto, mantener el control sobre el orden social, restringir la venta de sexo a ciertos sectores de la sociedad, controlar el contagio de enfermedades venéreas y delimitar el número y prácticas de las trabajadoras sexuales (Wagenaar, Altink y Amesberger, 2013).

Austria, Bélgica y Grecia han aplicado este modelo jurídico para regular el comercio sexual. En Austria, por ejemplo, es obligatorio el registro de las trabajadoras sexuales (aunque el procedimiento se ve modificado en cada región) y un chequeo médico semanal que, dependiendo de los resultados, posibilita o prohíbe la venta de sexo, entre otras disposiciones (Wagenaar, Altink y Amesberger, 2013). De acuerdo con Elvira Villa Camarma (2010), la reglamentación podría resultar, en algunos casos, coercitiva respecto de algunos derechos civiles como viajar, tener hijos, tener acceso a cobertura social y de salud para su familia, entre otros aspectos.

Finalmente, el modelo laborista busca que las trabajadoras sexuales puedan contar con la misma protección social y derechos laborales que cualquier otra trabajadora, equiparando el comercio sexual a otras prácticas laborales aceptadas, reguladas y protegidas en derechos. Pocos son los países que han decidido implementar este sistema de regulación, entre los que destaca Alemania. No obstante, las trabajadoras sexuales alemanas han relevado la importancia de la correcta implementación de este modelo con el fin de no resultar un sistema que, en la práctica, mantenga un alto porcentaje de prostitución clandestina. Explican que "La vía correcta sería descriminalizar la industria sexual, introducir cambios legislativos para calificar la prostitución como una forma de trabajo en vez de tratar de deshacerse de la industria con restricciones. La forma actual dejará solo dos variantes — quedarse en la sombra, trabajando ilegalmente, o ingresar en un gran burdel capaz de cumplir con tales normas" (Sputnik mundo, 25-03-16).

Ahora bien, no todos los Estados han llevado a cabo la discusión y resolución jurídica en materia de venta de servicios sexuales, por lo que la implementación de estos modelos no siempre se lleva a cabo a cabalidad. Es posible apreciar esta situación de modelos mixtos o ambiguos en Colombia (Tirado Acero, 2011), Uruguay (Musto y Trajtenberg, 2011), Chile (Fernández Darraz, 2011) y en una gran cantidad de países latinoamericanos (RedTraSex, 2018). Asimismo, en España el modelo jurídico que regula la transacción sexo/dinero no se ajusta a ninguno de los sistemas descritos (Brufao, 2008), no obstante, algunos autores analizan sus políticas públicas como tendientes al abolicionismo a causa de ciertas normativas que "sancionan el proxenetismo y considera la prostitución como un ejercicio amoral” (Santoyo Salgado, 2016: 40). Así, la realidad jurídica de cada uno de los Estados dependerá no solo del modelo que decidan emplear para regular el comercio sexual, sino que, antes de eso, de aquellas prácticas normativas y punitivas que establezcan frente a este fenómeno y que revelen cuáles son las retóricas que hay detrás de los documentos jurídicos concretos.

Para hablar de violencia hacia las mujeres en la industria del sexo es necesario, entonces, 
posicionarse en una mirada que permita la consideración de los múltiples factores expuestos en este artículo. Inicialmente se vuelve imprescindible nutrir la acotada mirada que polariza a quienes venden sexo en una sola categoría limitada y estricta (ya sea como mujer empoderada, víctima determinada o sujeto criminal); frente a esto, se vuelve fundamental la aceptación de la existencia de una realidad compleja en la que cohabitan tanto las personas oprimidas y determinadas por su entorno a vender servicios sexuales como quienes lo hacen voluntaria y libremente como una práctica laboral; asimismo, es importante suprimir aquella perspectiva que criminaliza el comercio sexual puesto que, ya sea de manera voluntaria u oprimida, las personas involucradas no se convierten en 'sujetos criminales' sino que en 'víctimas' o 'trabajadoras' de acuerdo con el contexto en que lleven a cabo estas prácticas.

La aceptación de este paradigma múltiple o polymorphus, como lo denomina Weitzer (2009) permite comprender no solo la situación de opresión/empoderamiento de las personas que venden servicios sexuales, sino que también la participación de una amplia gama de relaciones de poder, modelos jurídicos, acuerdos laborales, experiencias de los trabajadores, plataformas de ventas, tipos de servicios, entre otros elementos que se relacionan a la industria. "A diferencia de los otros dos, este paradigma es sensible a las complejidades y a las condiciones estructurales que configuran la distribución desigual de la agencia, la subordinación y la satisfacción laboral”8 (Weitzer, 2009: 215).

Es importante destacar la responsabilidad que tienen los Estados como delimitador del marco legislativo bajo cuyas disposiciones se inserta la industria sexual. De estas se desprenden múltiples consecuencias para la práctica concreta de llevar a cabo una transacción sexo/dinero: la existencia y delimitación de espacios exclusivos de venta de servicios sexuales (como burdeles o barrios); la penalización o no de los compradores o administradores de estos servicios; el cuidado médico y seguridad social a disposición de quienes venden sexo; la delimitación de plataformas de transacción (prostíbulos, cafés, Internet, llamadas, hoteles, calles, etc.), entre otras variables que pueden afectar el ejercicio de esta industria.

Por otra parte, como lo menciona Tirado Acero (2011: 145),

“[...] el Estado, frente a su responsabilidad como garante de la protección de los derechos sociales, económicos y culturales conexos con los derechos fundamentales, está obligado a proporcionar la protección adecuada a los miembros de la sociedad, con fundamentos en todas aquellas disposiciones de tipo constitucional".

Es, por lo tanto, fundamental la reflexión en torno a las políticas públicas aplicadas respecto de la industria del sexo y a cómo estas establecen relaciones de opresión/violencia o bien protección/seguridad para las mujeres involucradas en la venta de servicios sexuales.

\footnotetext{
${ }^{8}$ Traducción propia del original: "Unlike the other two, this paradigm is sensitive to complexities and to the structural conditions shaping the uneven distribution of agency, subordination, and job satisfaction".
} 


\section{Contexto jurídico y comercio sexual en Chile}

En Chile no existe un marco jurídico definido que regule el comercio sexual y, por lo tanto, no es posible dar cuenta de una retórica clara respecto de la posición desde la que el Estado enfrenta este fenómeno y su desarrollo. El análisis del contexto político en el que se inserta la industria del sexo en Chile cobra relevancia en tanto afecta directamente las condiciones de bienestar de las personas que venden sexo.

Actualmente son tres los documentos legislativos que se refieren a la compra/venta de servicios sexuales en el país: el Código Penal, el Código Sanitario y el Reglamento sobre Infecciones de Transmisión Sexual. A continuación se realiza una breve descripción y análisis de estos documentos y de su responsabilidad ante la protección social, médica, policial y laboral de las personas que venden sexo en el país.

En primer lugar, el Código Penal no alude directamente a la venta de servicios sexuales de manera consensuada y en libertad, sino que, en el artículo 367, penaliza la promoción o facilitación de la "prostitución de menores de edad para satisfacer los deseos de otro". El comercio sexual no se considera explicita y concretamente un delito en la regulación chilena, pero tampoco se contempla, en este Código, una referencia directa a esta industria, sino solo en tanto transgresión sexual de un menor ${ }^{9}$.

El Código Sanitario, por su parte, no utiliza la palabra "prostitución” para hacer mención a la venta de sexo en Chile, sino que establece las disposiciones sanitarias para el "comercio sexual": "Un reglamento establecerá [...] las condiciones en que se podrá examinar, obligar a tratarse o internar para su curación a las personas que se dediquen al comercio sexual y a las que estén afectadas de males venéreos que constituyan una amenaza para la salud pública" (Código Sanitario 1967: art 39). Al no utilizar el término "prostituta", distancia su postura del prohibicionismo, que criminaliza la figura de las mujeres que venden sexo. Por su parte, el Reglamento sobre Infecciones de Transmisión Sexual, emitido el año 2007 por el Ministerio de Salud, utiliza las palabras "trabajadores (as) sexuales" y "ejercicio del comercio sexual" para referirse a la venta de sexo.

La elección de los términos utilizados en los documentos jurídicos dan cuenta de los enfoques con que se intenta regular el comercio sexual: se evidencia el reconocimiento estatal de una industria de carácter sexual en Chile y de la no criminalización de esta; asimismo, se reconoce

\footnotetext{
${ }^{9}$ Es importante aquí diferenciar tres contextos de la venta de sexo: primero, aquella que se lleva a cabo de manera voluntaria por una persona mayor de edad; en segundo lugar, la venta de servicios sexuales de un tercero menor de edad (penalizado por el Código Penal chileno); finalmente, la trata de personas con fines de explotación sexual. El tráfico ilícito de personas con fines de explotación (entre los que se considera la prostitución) es una práctica delictual penada internacionalmente por el "Protocolo para Prevenir, Reprimir y Sancionar la Trata de Personas, especialmente Mujeres y Niños". Este tratado fue incorporado jurídicamente en Chile, según Decreto $N^{\circ} 342$ del Ministerio de Relaciones Exteriores, Publicado en Diario Oficial el 16 de febrero de 2005. Disponible en: http://bcn.cl/3wr4 (Junio, 2014) [12/05/2019].
} 
la necesidad de educación sexual, cuidado sanitario y tratamiento médico de quienes participan de la venta de servicios sexuales.

No obstante, los documentos jurídicos entran en tensión con las políticas públicas en términos de prácticas concretas. Pese a reconocer el "trabajo sexual" y a la "trabajadora sexual", no se regula estatalmente esta industria bajo una mirada de derechos laborales, sino que solo en tanto participan de ella "personas con conductas de riesgo" (Reglamento sobre Infecciones de Transmisión Sexual 2007: art. 9) para la salud sexual de la sociedad. Dejando, de este modo, a las "trabajadoras sexuales" relegadas a un espacio comercial irregular y sin protección social.

El artículo 38 del mismo Código establece el marco contextual en el que se habla del comercio sexual: "El Servicio Nacional de Salud tendrá a su cargo la lucha contra las enfermedades venéreas”, estableciendo, a través de este preludio el enfoque de las políticas públicas que regulan el comercio sexual: un problema sanitario.

Para hacer frente a este problema, se disponen para las trabajadoras sexuales controles médicos, tratamientos (obligatorios) en caso de enfermedades venéreas y la posibilidad de acceder "un documento que acredite su número de registro y asistencia a controles" (Reglamento sobre Infecciones de Transmisión Sexual 2007: art. 9).

Respecto de las regulaciones en las prácticas del comercio sexual, el mismo Código Sanitario prohíbe "su agrupación en prostíbulos cerrados o casas de tolerancia" (art. 41), inscribiendo la venta de sexo en una actividad privada e independiente. A través de este acto regulatorio, y de las disposiciones sanitarias, la mirada jurídica en Chile tiende al abolicionismo. Tal y como explican Sáez Tapia y Aravena Canales:

\footnotetext{
"Esta norma marca la tendencia legislativa abolicionista que ha adoptado Chile en relación a la prostitución, puesto que si bien se enmarca en un contexto de una política de salud del Estado, es posible advertir que se busca disuadir la organización de las personas que ejercen el comercio lascivo, ya que se considera esta actividad como peligrosa para la salud de las personas, de manera que les impone un control de salud obligatorio, con el objeto de llevar una estadística sanitaria. La infracción a esta disposición permite la clausura de dichos locales, y faculta al Servicio Nacional de Salud para imponer otro tipo de sanciones" (en Rivera Restrepo, 2007: 383).
}

Esta mirada reconoce el comercio sexual como una realidad social y económica, no lo juzga como un acto delictivo y entrega servicios de control y tratamientos médicos (con fines anti venéreos). No obstante, esta tendencia abolicionista del marco jurídico chileno lleva a la sistemática invisibilización de la realidad compleja de la industria sexual en Chile. Se reduce la venta de sexo a los formatos tradicionales y se omiten las necesidades sociales concretas de las personas que encuentran en la industria del sexo su principal fuente de ingreso económico.

La cuantificación e información estadística adecuada que permita la generación de políticas públicas contextualizadas a la realidad de las trabajadoras sexuales no existe. De acuerdo con el 
Reglamento sobre Infecciones de Transmisión Sexual "El establecimiento de salud que brinde atención de salud sexual a trabajadores(as) sexuales, deberá llevar una estadística sanitaria del número de personas en control de manera periódica" (2007: art. 10). Sin embargo, al ser los controles médicos voluntarios y confidenciales, la información recopilada carece de representatividad respecto del número total de trabajadores sexuales en el país. Por su parte, no todas las personas que venden sexo deciden recibir servicios de salud de los organismos públicos a causa de la discriminación y estigmatización de su labor. En la "Guía de buenas prácticas en el personal del sistema de salud", las trabajadoras sexuales exponen que en América Latina y el Caribe, aproximadamente un $75 \%$ utiliza el sistema público de salud y que "no somos pocas las que nos hemos sentido discriminadas" (RedTraSex, 2013: 11).

A partir de esto es posible decir que el marco jurídico chileno que regula el comercio sexual se constituye como un tejido de prácticas estatales que violentan de manera sistemática a las personas que venden sexo. La ausencia de información estadística ajustada a la realidad, la invisibilización de los problemas concretos de la venta de servicios sexuales y la ausencia de políticas públicas que entreguen a las trabajadoras sexuales estabilidad y seguridad social, laboral y policial dan cuenta de que, a pesar de la preocupación sanitaria inicial, el comercio sexual se mantiene inscrito en un espacio de marginalidad e invisibilización.

\section{Violencia institucional sobre las personas que venden sexo}

La tendencia abolicionista del marco jurídico actual en Chile se encuentra dado principalmente por tres hechos: el reconocimiento y la no criminalización de quienes venden sexo, la prohibición de una industria organizada en torno a la venta de servicios sexuales y el cuidado médico y sanitario puesto a disposición de las trabajadoras sexuales. Ahora bien, a pesar de que las retóricas abolicionistas consideran que la industria del sexo es fundamentalmente violenta en tanto reproduce y permite la explotación sexual de las mujeres (Lipszyc, 2003; Juliano, 2002; Barry, 1987), la invisibilización y ausencias en el modelo jurídico chileno pueden analizarse también como un perpetuador de violencia institucional hacia las trabajadoras del sexo (Sanders y Campbell, 2007; Scoular y O’Neill, 2007).

La violencia institucional se caracteriza por la participación activa de tres elementos: prácticas concretas, funcionarios públicos y contextos de restricción y autonomía de libertad. Es decir, se trata de "prácticas estructurales de violación de derechos por parte de funcionarios pertenecientes a fuerzas de seguridad, fuerzas armadas, servicios penitenciarios y efectores de salud en contextos de restricción de autonomía y/o libertad (detención, encierro, custodia, guarda, internación, etc.)" (Ministerio de Justicia y Derechos Humanos, 2012: 13). Por su parte, la aplicación de estas violencias puede ser llevada a cabo por acción o por omisión; en el primer caso, 
los funcionarios ejecutan prácticas concretas que violentan los derechos humanos y, en el segundo, los funcionarios dejan de cumplir con el rol que les ha sido asignado y que asegura el resguardo de los derechos de los ciudadanos (RedTraSex, 2018).

Las agrupaciones de mujeres que venden servicios sexuales, a pesar de su carácter marginal, han creado esferas (Fraser, 1997) en las que es posible insertar su discurso, sus perspectivas y sus demandas. Por medio de la divulgación de información que permiten estas esferas, se han dado a conocer las principales causas de violencia institucional por acción. La Red de Mujeres Trabajadoras Sexuales de Latinoamérica y el Caribe realizó un estudio titulado Estudio sobre Situaciones de Violencia Institucional hacia Mujeres Trabajadoras Sexuales y Vulneración de Derechos (2016) en el que participaron trabajadoras sexuales de 14 países de Latinoamérica y cuyos resultados se organizaron en un informe regional y un informe específico para cada país.

En un estudio posterior, realizado el año 2018 también a nivel latinoamericano, de las más de 5.000 mujeres trabajadoras sexuales encuestadas, el 73.7\% (3.813) declaran haber sufrido violencia por parte de las fuerzas de seguridad de su país. De estos tipos de violencias efectuados por las fuerzas de seguridad en Latinoamérica y el Caribe, se destacan la violencia verbal, psicológica, física, sexual y económica (a través de extorsiones y coimas) (RedTraSex, 2018).

\footnotetext{
"Este último aspecto es clave para comprender a la violencia institucional y policial como violencia de género: las MTS son abusadas, violadas o intimidadas sexualmente en virtud de la naturalización de una doble disponibilidad sexual: están disponibles por su condición de mujeres y lo están doblemente por hacer de las prácticas sexuales una herramienta laboral" (RedTraSex, 2018: 19).
}

El informe nacional del año 2016 (realizado por la RedTraSex en conjunto con la Fundación Margen) arrojó resultados respecto de las prácticas de violencia institucional a las que se ven enfrentados quienes venden servicios sexuales en Chile (RedTraSex, 2016). Respecto de las violencias por acción, las mujeres declaran haber sufrido detenciones arbitrarias por parte de las fuerzas policiales, en cuyos procedimientos las violentan a través de "constantes amenazas físicas, psicológicas y sexuales propinadas" (RedTraSex, 2016: 8), de solicitud de servicios sexuales gratuitos, humillaciones, entre otras prácticas. Por su parte, en la "Guía de buenas prácticas en el personal del sistema de salud" (2013) perteneciente a la misma institución, se da cuenta de las vulneraciones de derechos a través de la falta de confidencialidad, uso de términos descalificatorios, ser tratadas como foco de infección, entre otras prácticas de quienes participan del sistema de salud público.

Respecto de las violencias institucionales por omisión, se establecen en el presente texto, la existencia de dos grandes aristas: en primer lugar, el aspecto judicial que deliberadamente ignora el conflicto de violencias involucradas en el comercio sexual y se desenmarca de este. Así, las mujeres trabajadoras sexuales "expresan que no tienen relación con jueces, fiscales, ni defensores" (RedTraSex, 2016: 15) y que solamente reconocen como funcionarios judiciales a los fiscalizadores 
municipales. En esta primera arista, los jueces o fiscales que deberían representar una figura a la que acudir en caso de agresiones policiales o de vulneración de derechos, se transforman en seres etéreos del sistema frente a quienes las mujeres "prefieren optar por el silencio, debido a que consideran que no serán escuchadas ni apoyadas por la justicia por efectuar el trabajo sexual" (RedTraSex, 2016: 15).

La segunda arista de las violencias por omisión se encuentra justamente en el vacío jurídico en el que se encuentran las personas que venden sexo de manera deliberada y voluntaria en el país. La ausencia de su reconocimiento laboral y de la protección social posiciona a las trabajadoras sexuales en un espacio de vulnerabilidad y ausencia que las violenta (Sanders y Campbell, 2007; Scoular y O’Neill, 2007, Gira Grandt, 2016). Quienes participen de la industria del sexo en Chile, por lo tanto, se encuentran expuestos no solo al complejo escenario que significa la venta de sexo en sí misma (su construcción, simbolización, estigmatización sanitaria y social, peligrosidad, entre otros factores), sino que también a la violencia institucional a causa de los abusos de poder y de la invisibilización de este cuerpo social.

\section{Conclusiones}

Las violencias a las que se encuentran sometidas las personas que venden sexo son múltiples. Para su análisis es necesario considerar los distintos focos de violencia y el funcionamiento concreto de estos. La perspectiva bajo la que se ha llevado a cabo su construcción histórica, los paradigmas que construyen la imagen de quienes venden sexo desde la opresión/empoderamiento, los modelos de regulación jurídica en los que se enmarca, la realidad socio-laboral compleja y precaria del comercio sexual, se configuran como los principales escenarios de violencia en la industria.

Los espacios y los contextos del ejercicio de la venta de sexo son variados y dinámicos, adaptándose a los contextos, necesidades, ley de oferta/demanda, valores de los servicios, plataformas, entre otras variables. Las miradas desde las que se analiza el comercio sexual son, por lo general, binarias y se posicionan desde polaridades que no abarcan la multiplicidad y complejidad (teórica, económica, social, política y sexual) que significa el comercio sexual y la mercantilización del deseo sexual. Por su parte, los modelos jurídicos no se ajustan a las necesidades y complejidades del fenómeno. Particularmente en Chile, el modelo genera instancias de cuidado y control médico (en tanto problema sanitario y con fines anti venéreos), pero invisibiliza a quienes venden sexo en todos los otros aspectos.

Estas condiciones determinan la posición de las trabajadoras sexuales en un espacio de vulnerabilidad e inseguridad provocando dos efectos principales: en primer lugar, el aumento de los riesgos propios del trabajo sexual (marginalidad, violencia, precarización de los espacios laborales, abuso de poder de parte de los proxenetas, entre otros); y, en segundo lugar, el ejercicio de violencia 
en la invisibilización de sus derechos laborales y protección social.

La importancia de comprender las violencias que se circunscriben en las políticas públicas de cada país y que afectan a las mujeres que participan de la industria del sexo radica en la generación de marcos jurídicos que otorguen niveles mínimos de protección, estabilidad y seguridad para ellas. Así lo comprende Elvira Villa Camarma al explicar que:

“[...] el derecho laboral fue concebido como una fórmula de protección para los trabajadores. Por tanto, al elevar a estas mujeres a esta categoría las estamos dotando de un armamento legal con que defenderse, tanto de empresarios como de clientes y de mafias; sin victimizarlas ni infantilizarlas" (Villa Camarma, 2010: 176).

A partir de este análisis, es posible establecer que, por una parte, el modelo jurídico chileno tiende a una mirada que reconoce a quienes venden sexo como una víctima de un sistema de explotación; no obstante, por otra parte, se encuentra en contradicción consigo mismo al perpetuar un sistema de explotación en el que las mujeres no poseen herramientas judiciales, espacios de protección policial o estabilidad económica y social. Los modelos jurídicos adoptados por el Estado tienen la posibilidad de transformarse en políticas de protección para las trabajadoras sexuales y, de este modo, asegurar y delimitar prácticas seguras en la venta de sexo, disminuyendo los focos de violencia que afectan a esta industria. No obstante, en la actualidad, la presencia de las mujeres que venden servicios sexuales como cuerpo social se encuentra reducido a la posibilidad de atención médica e invisibilizado en términos de derechos generando, de esta manera, mayores niveles de violencias para las mujeres que participan de esta actividad comercial.

\section{BibLIOGRAFÍA}

- Araya Ibache, Claudia (2006): "La construcción de una imagen femenina a través del discurso médico ilustrado. Chile en el siglo XIX”. En: Revista Historia, vol. 1, nº. 39, pp. 5-22.

- Barry, Kathleen (1987): La esclavitud sexual de la mujer. Barcelona: LaSal edicions de les dones.

- Bell, Shannon (1994): Reading, Writing and Rewriting the prostitute body. Indiana: Indiana University Press.

- Bolaños Naranjo, Alicia (2008): "La prostitución desde una perspectiva de los derechos humanos" [Conferencia] Concepciones y posicionamientos sobre la prostitución.

- Brufao, Pedro (2008): Prostitución y políticas públicas: entre la reglamentación, la legalización y la abolición. España: Fundación Alternativas. Disponible en: https://dialnet.unirioja.es/servlet/libro?codigo=290918 [14/05/2019]. 
- Canales Nettle, Patricia (2005): “La regulación de la prostitución en legislación comparada". En: Biblioteca del Congreso Nacional de Chile, pp. 1-33.

- Díez Gutiérrez, Enrique (2009): “Prostitución y violencia de género”. En: Nómadas. Critical Journal of Social and Juridical Sciences, vol. 24, nº 4, pp. 1-4.

- Dodillet, Susanne y Östergren, Petra (2011): "La Ley de compra de sexo sueca: éxito proclamado y resultados documentados", pp. 1-36. [Conferencia] Presentado en Taller internacional: Despenalización de la prostitución y más allá: experiencias prácticas y retos, La Haya.

- Durán, Manuel (2009): "Medicalización y disciplinamiento. La construcción higienista del espacio femenino, 1850-1920”. En: Nomadías, n. 9, pp. 123-139.

- Fernández Darraz, Ma Cecilia (2011): "El comercio sexual en Chile: ambiguedades y contradicciones discursivas", En: Anagramas, n. 9, pp. 71-82.

- Folchi Donoso, Mauricio (2007): "La higiene, la salubridad pública y el problema de la vivienda popular en Santiago de Chile (1843-1925)". En: Perfiles habitacionales y condiciones ambientales. Historia Urbana de Latinoamérica siglos XVII-XX. México DF: Benemérita Universidad Autónoma de Puebla, pp. 361-388.

- Foucault, Michel (2003): Hay que defender la sociedad. Curso del Collège de France (19751976). Madrid: Ediciones Akal.

. (2012): Vigilar y Castigar. Nacimiento de la prisión (2 ${ }^{\mathrm{a}}$ edición). Buenos Aires: Siglo veintiuno editores.

- Gálvez Comandini, Ana (2014): "Lupanares, burdeles y casas de tolerancia: tensiones entre las prácticas sociales y la reglamentación de la prostitución en Santiago de Chile: 1896-1940”. En: Revista Tiempo Histórico, nº. 8, pp. 73-92.

- Gira Grant, Melissa. (2016): Haciendo de puta. La labor del trabajo sexual (1. a ed.). Santiago: Pólvora.

- Góngora Escobedo, Álvaro (1999): La prostitución en Santiago, 1813-1931: visión de las elites. Santiago de Chile: Editorial Universitaria.

- Gould, Arthur (2002): “La ley sueca sobre prostitución”. En: Revista de treball social, n. 167, pp. 6-23.

- Licht, Hans (2009): Sexual life in Ancient Greece. New York: Routledge.

- Lipszyc, Cecilia (2003): "Mujeres en situación de prostitución: ¿esclavitud sexual o trabajo sexual?”. En: Prostitución: ¿trabajo o esclavitud sexual? Lima: Comité deAmérica Latinay el Ca ribepara la Defensa de los Derechos de la Mujer-CLADEM, pp. 55-70.

- Maira, Octavio (1887): La reglamentación de la prostitución desde el punto de vista de la higiene pública (Grado de Licenciado en la Facultad de Medicina y Farmacia). Universidad de Chile, Santiago de Chile.

- Ministerio de Justicia y Derechos Humanos (2012): Los derechos humanos frente a la Violencia Institucional. Argentina. 
- Monje Álvarez, Carlos Arturo (2011): Metodología de la Investigación Cualitativa y Cuantitativa. Universidad Sur Colombia, Facultad de Ciencias Sociales y Humanas.

- Musto, Clara y Trajtenberg, Nico (2011): "Prostitución y trabajo sexual: el estado de arte de la investigación en Uruguay”. En: Revista de Ciencias Sociales, vol. 24, nº. 29, pp. 138-156.

- Oficina Internacional del Trabajo (1998): "La industria del sexo: los incentivos económicos y la penuria alimentaria alimentan el crecimiento". Octubre. En: Revista Trabajo, no. 26, pp. 10-14.

- OMS - Organización Mundial de la Salud (2013): Estimaciones mundiales y regionales de la violencia contra la mujer: Prevalencia y efectos de la violencia conyugal y de la violencia sexual no conyugal en la salud. Ginebra.

- Pateman, Carol (1995): El contrato sexual. Barcelona: Anthropos.

- RedTraSex (2013): Estudio sobre estigma y discriminación en los servicios de salud a las mujeres trabajadoras sexuales en América Latina y el Caribe (apoyo del Fondo Mundial de Lucha contra el Sida, la Tuberculosis y la Malaria.).

. (2013). “«Ponte en nuestros zapatos» Guía de buenas prácticas en el personal del sistema de salud", 10 de diciembre. Disponible en: http://www.redtrasex.org/Guia-de-buenas-practicas-enel.html [12/05/2019].

. (2016): "Trabajo sexual y violencia institucional: vulneración de derechos y abuso de poder hacia las mujeres trabajadoras sexuales". Estudio sobre Situaciones de Violencia Institucional hacia Mujeres Trabajadoras Sexuales y Vulneración de Derechos. Chile: Red de Mujeres Trabajadoras Sexuales de Latinoamérica y el Caribe. Disponible en: http://www.redtrasex.org/Trabajo-sexual-yviolencia.html [12/05/2019].

(2016): "Violencia Institucional hacia Trabajadoras sexuales. Informe Nacional Chile" (Estudio sobre Situaciones de Violencia Institucional hacia Mujeres Trabajadoras Sexuales y Vulneración de Derechos). Chile: Fundación Margen, p. 22. Disponible en: http://www.redtrasex.org/Trabajo-sexual-y-violencia.html [12/05/2019].

(2018): El trabajo sexual y la violencia institucional. Cuando la clandestinidad va de la mano del abuso de autoridad y de la vulneración de derechos (Estudio sobre Situaciones de Violencia Institucional hacia Mujeres Trabajadoras Sexuales y Vulneración de Derechos). Buenos Aires: Red de Mujeres Trabajadoras Sexuales de Latinoamérica y el Caribe, p. 44.

- Ribeiro, Manuela y Sacramento, Octavio (2005): "Violence against Prostitutes. Findings of Research in the Spanish-Portuguese Frontier Region". En: European Journal of Women's Studies, $\mathrm{n}^{\mathrm{o}}$. 12, pp. 61-81. Disponible en: https://journals.sagepub.com/doi/10.1177/1350506805048856 [12/05/2019].

- Rivera Restrepo, José (2017): “Algunos apuntes jurídicos sobre la prostitución en Chile”. Boletín Mexicano de Derecho Comparado, no. 148, pp. 361-392.

- Sanders, Teela y Campbell, Rosie (2007): "Designing out vulnerability, building in respect: violence, safety and sex work policy". En: The British Journal of Sociology, n. 58, pp. 1-19. 
- Sanders, Teela; O’Neill, Maggie y Pitcher, Jane (2018): Prostitution. Sex Work, Policy \& Politics (2nd edition). London: SAGE.

- Sanger, William (1958): The history of prostitution. New York: Harper and Brothers.

- Scoular, Jane y O’Neill, Maggie (2007): “Regulating Prostitution. Social Inclusion, Responsibilization and the Politics of Prostitution Reform”. En: The British Journal of Criminology, no. 47, pp. 764-778. https://academic.oup.com/bjc/article/47/5/764/459991 [12/05/2019].

- Sex Workers Alliance Ireland (2017): Sex Workers Alliance Ireland updated shadow report information for CEDAW. Ireland: Sex Workers Alliance Ireland, p. 5.

- Tirado Acero, Misael (2011): "El debate entre prostitución y trabajo sexual. Una mirada desde lo socio-jurídico y la política pública”. En: Revista de Relaciones Internacionales, Estrategia y Seguridad, no. 6, pp. 127-148.

- Urriola Pérez, Ivonne (1999): "Espacio, oficios y delitos femeninos: el sector popular de Santiago 1900-1925”. En: Historia, no. 32, pp. 443-483.

- Vera Gajardo, Antonieta (2016): "La superioridad moral de la mujer: sobre la norma racializada de la femenidad en Chile”. En: Historia y Política, no. 36, pp. 211-240.

- Vera Gutiérrez, Marlene (2018): "Las prostitutas y sus discursos: voces que emergen desde los archivos. Santiago 1880-1925”. Magister en estudios de género y cultura, mención humanidades. Universidad de Chile, Santiago de Chile.

- Villa Camarma, Elvira (2010): “Estudio antropológico en torno a la prostitución”. En: Cuicuilco, vol. $17, \mathrm{n}^{\circ} .49$, pp. 157-179

- Wagenaar, Hendrik; Altink, Sietske y Amesberger, Helga (2013): Final Report of the International Comparative Study of Prostitution Policy: Austria and the Netherlands. La Haya: Plataform 31.

- Weitzer, Ronald (2009): “Sociology of Sex Work". En: Annual Review of Sociology, n. 35, pp. 213-234. Disponible en: https://www.annualreviews.org/doi/10.1146/annurev-soc-070308-120025 [12/05/2019].

- Wells, Jess (1982): Herstory of Prostitution in Western Europe (First edition). Berkeley, C.A.: Shameless Hussy Pr.

- Zárate, Ma Soledad (1995): "Mujeres viciosas, mujeres virtuosas. La mujer delincuente y la Casa Correccional de Santiago 1860-1900". En: Disciplina y desacato. Construcción de identidad en Chile siglos XIX y XX. Santiago de Chile: SUR, pp. 149-180.

\section{Periódicos online}

- Sputnik mundo (2016): "Prostitutas alemanas quieren abortar una nueva leye que viola sus derechos", 25 de marzo. Disponible en: https://sptnkne.ws/ddKG [12/05/2019].

- Sputnik mundo (2017): "Prostitutas de París protestan contra la leyes que castiga a sus clientes", 08 de abril. Disponible en: https://sptnkne.ws/eb43 [12/05/2019]. 